PROCEEDINGS OF THE

AMERICAN MATHEMATICAL SOCIETY

Volume 136, Number 8, August 2008, Pages 2881-2886

S 0002-9939(08)09238-1

Article electronically published on April 8, 2008

\title{
A NOTE ON BILINEAR ESTIMATES AND REGULARITY OF FLOW MAPS FOR NONLINEAR DISPERSIVE EQUATIONS
}

\author{
SEBASTIAN HERR
}

(Communicated by Hart F. Smith)

\begin{abstract}
Explicit counterexamples to bilinear estimates related to the Benjamin-Ono equation in the periodic setting are calculated for functions of zero mean value. As a consequence, certain bilinear estimates fail to hold in spite of the analyticity of the flow map. The latter has been shown recently by L. Molinet.
\end{abstract}

\section{INTRODUCTION AND MAIN RESULT}

A common approach to local well-posedness problems for nonlinear dispersive partial differential equations is based on the contraction mapping principle applied to a corresponding integral operator. The key to such arguments are nonlinear estimates which establish the contraction property. This method necessarily leads to analytic dependence of the solution on the initial data, provided that the nonlinearity is analytic. The works of J. Bourgain [1] and C. E. Kenig, G. Ponce and L. Vega [5, 6] are prominent examples for a successful implementation of this general strategy in connection with the Korteweg-de Vries equation. However, it turned out that there are well-posed problems with a continuous flow map, such as the Benjamin-Ono equation with initial data in the Sobolev spaces $H^{s}(\mathbb{R})$; see e.g. [11, 4], where multilinear estimates fail to hold true, and see [8]. The counterexamples from 8 are closely connected with nonsmoothness of the flow map; cf. [7.

In this note we show that the failure of multilinear estimates does not in general imply regularity restrictions on the flow map. We consider the Cauchy problem for the Benjamin-Ono equation

$$
\begin{aligned}
\partial_{t} u-\mathcal{H} \partial_{x}^{2} u & =\partial_{x}\left(u^{2}\right) \quad \text { in }(0, T) \times \mathbb{T}, \\
u(0) & =u_{0} \in \dot{H}^{s}(\mathbb{T})
\end{aligned}
$$

where $\mathbb{T}=\mathbb{R} / 2 \pi \mathbb{Z}$ and $\dot{H}^{s}(\mathbb{T}):=\left\{u: \mathbb{T} \rightarrow \mathbb{R} \mid u \in H^{s}(\mathbb{T}), \int_{0}^{2 \pi} u d x=0\right\}$. Here, $\mathcal{H}$ denotes the Hilbert transform, defined by $\widehat{\mathcal{H} u}(k)=-i \operatorname{sign}(k) \widehat{u}(k)$. We restrict

Received by the editors April 17, 2007.

2000 Mathematics Subject Classification. Primary 35Q53; Secondary 76B15, 35B30.

Key words and phrases. Failure of bilinear estimates, periodic Benjamin-Ono equation.

The author is grateful to M. Hadac, H. Koch, and N. Tzvetkov for valuable discussions on the subject. This research is part of the author's doctoral dissertation, which has been awarded a Dissertationspreis at Dortmund University.

(C)2008 American Mathematical Society 2881

Reverts to public domain 28 years from publication 
ourselves to initial data with zero mean and remark that the mean value is a conserved quantity for solutions of (1.1).

In his recent works [9, 10, L. Molinet gradually proved global well-posedness of the Benjamin-Ono equation in $H^{\frac{1}{2}}(\mathbb{T})$ and in $L^{2}(\mathbb{T})$. These results include Lipschitz continuity and moreover the real analyticity of the flow map $u_{0} \mapsto u$ on balls containing initial data with vanishing mean value. His approach is based on a gauge transformation, cf. [11, and subsequent multilinear estimates for the transformed problem. For the precise statements we refer the reader to [9, 10]. Here, we will complement these results with a counterexample which reveals the importance of using the gauge transformation: In spite of the underlying real analyticity of the flow map it is impossible to prove reasonable (in the sense of Theorem 1.1) bilinear estimates directly.

We know that the flow map is not uniformly continuous on balls $B_{R}(0) \subset H^{s}(\mathbb{T})$ for any $s>0, R>0$ for initial data with arbitrary mean value; see [9]. This property is caused by the mean value $\mu$ of a solution, which leads to a linear transport term $\mu \partial_{x} u$ in the equation. In the nonperiodic case a corresponding phenomenon was shown in 7] (cf. [8] where it is shown that the flow map is not $C_{l o c}^{2}$ ), which is based on the interaction of two linear waves, one with frequencies near $N$ and one with frequencies near $1 / N$, for $N \rightarrow \infty$. This counterexample constitutes a severe obstruction for iterative methods to work. In fact, locally uniform continuity in the periodic setting even fails for equations with stronger dispersion such as the Korteweg-de Vries equation. However, for real-valued periodic functions the well-known transformation

$$
u(t, x) \mapsto u\left(t, x-t \frac{1}{2 \pi} \int_{0}^{2 \pi} u(0, y) d y\right)-\frac{1}{2 \pi} \int_{0}^{2 \pi} u(0, y) d y
$$

maps solutions upon solutions and reduces the problem to zero mean value functions. This transformation was used in [1, 6, to treat the Korteweg-de Vries equation by iterative methods.

Now we state the main result of this note. Let $\dot{C}^{\infty}(\mathbb{T})$ be the linear space of all smooth functions $f: \mathbb{R} \rightarrow \mathbb{R}$ such that $f(t, x)=f(t, x+2 \pi)$ and $\int_{0}^{2 \pi} f(x) d x=0$. Similarly, let $\dot{C}^{\infty}([0, T] \times \mathbb{T})$ be the linear space of smooth functions $f:[0, T] \times \mathbb{R} \rightarrow$ $\mathbb{R}$ such that $f(t, \cdot) \in \dot{C}^{\infty}(\mathbb{T})$.

Theorem 1.1. Let $s \in \mathbb{R}, T>0$. There does not exist a normed space $X^{T}$ with $\dot{C}^{\infty}([0, T] \times \mathbb{T}) \subset X^{T}$ and $X^{T} \hookrightarrow C\left([0, T], \dot{H}^{s}(\mathbb{T})\right)$ and a constant $c>0$, such that the estimates

$$
\begin{gathered}
\forall u_{0} \in \dot{C}^{\infty}(\mathbb{T}): \quad\left\|e^{\mathcal{H} \partial_{x}^{2} t} u_{0}\right\|_{X^{T}} \leq c\left\|u_{0}\right\|_{H^{s}(\mathbb{T})} \\
\forall u \in \dot{C}^{\infty}([0, T] \times \mathbb{T}): \quad\left\|\int_{0}^{t} e^{\mathcal{H} \partial_{x}^{2}\left(t-t^{\prime}\right)} \partial_{x}\left(u\left(t^{\prime}\right)\right)^{2} d t^{\prime}\right\|_{X^{T}} \leq c\|u\|_{X^{T}}^{2}
\end{gathered}
$$

are valid.

This result is (in the spirit of [8], Theorem 1) a statement about the failure of techniques and not a statement about the regularity of the flow (such as [8], Theorem 2). It is antithetic to the case of the real line in the sense that bilinear estimates hold true in certain spaces of nonperiodic functions fulfilling an additional low frequency condition [3, 4] (cf. [2] concerning the dispersion generalized case). 
Remark 1.2. We remark without proof that Theorem 1.1 extends to the dispersion generalized equations

$$
\begin{aligned}
\partial_{t} u+|D|^{\alpha} \partial_{x} u & =\partial_{x}\left(u^{2}\right) \quad \text { in }(0, T) \times \mathbb{T}, \\
u(0) & =u_{0} \in \dot{H}^{s}(\mathbb{T})
\end{aligned}
$$

for $1 \leq \alpha<2$ with a similar proof by using the Taylor expansion of the phase function $\phi(\xi)=\xi|\xi|^{\alpha}$.

\section{Counterexamples And the Proof of Theorem 1.1}

We construct real-valued initial data with zero mean, such that a four-linear low-low-low-high interaction of linear waves provides a suitable estimate for the Duhamel term in $H^{s}(\mathbb{T})$ from below. Let

$$
B(u, v)(t)=\int_{0}^{t} e^{\mathcal{H} \partial_{x}^{2}\left(t-t^{\prime}\right)} \partial_{x}\left(u\left(t^{\prime}\right) v\left(t^{\prime}\right)\right) d t^{\prime}
$$

and for initial data $\psi_{N}$ define

$$
\begin{aligned}
& I_{1}=I_{1}\left(\psi_{N}\right)=e^{\mathcal{H} \partial_{x}^{2} t} \psi_{N}, I_{2}=I_{2}\left(\psi_{N}\right)=B\left(I_{1}\left(\psi_{N}\right), I_{1}\left(\psi_{N}\right)\right), \\
& I_{2,2}=I_{2,2}\left(\psi_{N}\right)=B\left(I_{2}\left(\psi_{N}\right), I_{2}\left(\psi_{N}\right)\right) .
\end{aligned}
$$

Lemma 2.1. For $s \in \mathbb{R}, N \in \mathbb{N}$ we define

$$
\psi_{N}(x):=\sqrt{\frac{2}{\pi}}\left(N^{-s} \cos (N x)-\cos (2 x)+\cos (x)\right) .
$$

Then, for all $t>0$ we have

$$
\left\|I_{2,2}\left(\psi_{N}\right)(t)\right\|_{H^{s}(\mathbb{T})} \geq N \sin ^{2}(t)-12 .
$$

Proof of Lemma 2.1. Without loss of generality we assume $N \geq 12$. The Fourier transform of $\psi_{N}$ is

$$
\widehat{\psi_{N}}(k)=\left\{\begin{aligned}
1, & & |k|=1 \\
-1, & & |k|=2, \\
N^{-s}, & & |k|=N
\end{aligned}\right.
$$

Since $e^{-\mathcal{H} \partial_{x}^{2} t}$ is an isometry,

$$
\left\|I_{2,2}(t)\right\|_{H^{s}(\mathbb{T})}=\left\|e^{-\mathcal{H} \partial_{x}^{2} t} I_{2,2}(t)\right\|_{H^{s}(\mathbb{T})} \geq N^{s}\left|e^{\mathcal{H} \partial_{x}^{2 t} I_{2,2}}(t)(N)\right|,
$$

and we take into account all interactions which contribute to the frequency $N$. Since $\widehat{I_{2}(t)}(0)=0$ we have to calculate $\widehat{I_{2}(t)}(k)$ for $|k|=1,|k|=2,|k-N|=1$, $|k-N|=2$ :

$$
\begin{aligned}
\widehat{I_{2}(t)}( \pm 1) & = \pm i 2 \int_{0}^{t} e^{ \pm i\left(t-t^{\prime}\right)}\left(-e^{ \pm 4 i t^{\prime}}\right) e^{\mp i t^{\prime}} d t^{\prime} \\
& =\mp i 2 e^{ \pm i t} \int_{0}^{t} e^{ \pm 2 i t^{\prime}} d t^{\prime} \\
& =-e^{ \pm i t}\left(e^{ \pm 2 i t}-1\right)
\end{aligned}
$$


and

$$
\begin{aligned}
\widehat{I_{2}(t)}( \pm 2) & = \pm 2 i \int_{0}^{t} e^{ \pm 4 i\left(t-t^{\prime}\right)} e^{ \pm i t^{\prime}} e^{ \pm i t^{\prime}} d t^{\prime} \\
& = \pm 2 i e^{ \pm 4 i t} \int_{0}^{t} e^{\mp 2 i t^{\prime}} d t^{\prime} \\
& =-e^{ \pm 4 i t}\left(e^{\mp 2 i t}-1\right) .
\end{aligned}
$$

Similarly we get

$$
\begin{aligned}
\widehat{I_{2}(t)}(N+1) & =2 i(N+1) \int_{0}^{t} e^{i\left(t-t^{\prime}\right)(N+1)^{2}} N^{-s} e^{i t^{\prime} N^{2}} e^{i t^{\prime}} d t^{\prime} \\
& =2 i(N+1) N^{-s} e^{i t(N+1)^{2}} \int_{0}^{t} e^{i t^{\prime}\left(N^{2}+1-(N+1)^{2}\right)} d t^{\prime} \\
& =-\frac{(N+1)}{N} N^{-s} e^{i t(N+1)^{2}}\left(e^{-2 N i t}-1\right),
\end{aligned}
$$

and

$$
\begin{aligned}
\widehat{I_{2}(t)}(N+2) & =2 i(N+2) \int_{0}^{t} e^{i\left(t-t^{\prime}\right)(N+2)^{2}} N^{-s} e^{i t^{\prime} N^{2}}(-1) e^{4 i t^{\prime}} d t^{\prime} \\
& =\frac{(N+2)}{2 N} N^{-s} e^{i t(N+2)^{2}}\left(e^{-4 N i t}-1\right),
\end{aligned}
$$

as well as

$$
\begin{aligned}
\widehat{I_{2}(t)}(N-1) & =2 i(N-1) \int_{0}^{t} e^{i\left(t-t^{\prime}\right)(N-1)^{2}} N^{-s} e^{i t^{\prime} N^{2}} e^{-i t^{\prime}} d t^{\prime} \\
& =2 i(N-1) N^{-s} e^{i t(N-1)^{2}} \int_{0}^{t} e^{i t^{\prime}\left(N^{2}-1-(N-1)^{2}\right)} d t^{\prime} \\
& =N^{-s} e^{i t(N-1)^{2}}\left(e^{(2 N-2) i t}-1\right),
\end{aligned}
$$

and

$$
\begin{aligned}
\widehat{I_{2}(t)}(N-2) & =2 i(N-2) \int_{0}^{t} e^{i\left(t-t^{\prime}\right)(N-2)^{2}} N^{-s} e^{i t^{\prime} N^{2}}(-1) e^{-4 i t^{\prime}} d t^{\prime} \\
& =-\frac{1}{2} N^{-s} e^{i t(N-2)^{2}}\left(e^{(4 N-8) i t}-1\right) .
\end{aligned}
$$

Next, we calculate the contribution to $N^{s} e^{\mathcal{H}\left(\widehat{\partial_{x}^{2} t} I_{2,2}\right.}(t)(N)$ from $\widehat{I_{2}\left(t^{\prime}\right)}(N-1)$ and $\widehat{I_{2}\left(t^{\prime}\right)}(1)$, where we use the relation $-N^{2}+1+(N-1)^{2}+2 N-2=0$. We have

$$
\begin{aligned}
J_{1} & :=2 \int_{0}^{t} e^{-i t^{\prime} N^{2}} i N(-1) e^{i t^{\prime}}\left(e^{2 i t^{\prime}}-1\right) e^{i t^{\prime}(N-1)^{2}}\left(e^{(2 N-2) i t^{\prime}}-1\right) d t^{\prime} \\
& =-2 i N\left(\int_{0}^{t}\left(e^{2 i t^{\prime}}-1\right) d t^{\prime}-\int_{0}^{t} e^{i t^{\prime}(2-2 N)}\left(e^{2 i t^{\prime}}-1\right) d t^{\prime}\right) \\
& =-2 i N\left(\frac{e^{2 i t}-1}{2 i}-t\right)+R_{1},
\end{aligned}
$$

with the bounded remainder term

$$
R_{1}:=-N\left(\frac{e^{i t(4-2 N)}-1}{N-2}-\frac{e^{i t(2-2 N)}-1}{N-1}\right)
$$


which satisfies $\left|R_{1}\right| \leq 5$. Similarly, because of $-N^{2}-1+(N+1)^{2}-2 N=0$ the contribution to $N^{s} e^{\mathcal{H}} \widehat{\partial_{x}^{2} t I_{2,2}}(t)(N)$ coming from $\widehat{I_{2}\left(t^{\prime}\right)}(N+1)$ and $\widehat{I_{2}\left(t^{\prime}\right)}(-1)$ is

$$
\begin{aligned}
J_{2} & :=2 i(N+1) \int_{0}^{t} e^{-i t^{\prime} N^{2}} e^{-i t^{\prime}}\left(e^{-2 i t^{\prime}}-1\right) e^{i t^{\prime}(N+1)^{2}}\left(e^{-2 N i t^{\prime}}-1\right) d t^{\prime} \\
& =2 i(N+1)\left(\int_{0}^{t}\left(e^{-2 i t^{\prime}}-1\right) d t^{\prime}-\int_{0}^{t} e^{2 N i t^{\prime}}\left(e^{-2 i t^{\prime}}-1\right) d t^{\prime}\right) \\
& =2 i(N+1)\left(\frac{e^{-2 i t}-1}{-2 i}-t\right)+R_{2},
\end{aligned}
$$

with the bounded remainder term

$$
R_{2}:=-(N+1)\left(\frac{e^{(2 N-2) i t}-1}{N-1}-\frac{e^{2 N i t}-1}{N}\right),
$$

which satisfies $\left|R_{2}\right| \leq 5$. Due to $-N^{2}+4+(N-2)^{2}+4 N-8=0$ the contribution to $N^{s} e^{\mathcal{H}} \widehat{\partial_{x}^{2} t I_{2,2}}(t)(N)$ coming from $\widehat{I_{2}\left(t^{\prime}\right)}(N-2)$ and $\widehat{I_{2}\left(t^{\prime}\right)}(2)$ amounts to

$$
\begin{aligned}
J_{3} & :=i N \int_{0}^{t} e^{-i t^{\prime} N^{2}} e^{4 i t^{\prime}}\left(e^{-2 i t^{\prime}}-1\right) e^{i t^{\prime}(N-2)^{2}}\left(e^{(4 N-8) i t^{\prime}}-1\right) d t^{\prime} \\
& =i N\left(\int_{0}^{t}\left(e^{-2 i t^{\prime}}-1\right) d t^{\prime}-\int_{0}^{t} e^{(-4 N+8) N i t^{\prime}}\left(e^{-2 i t^{\prime}}-1\right) d t^{\prime}\right) \\
& =i N\left(\frac{e^{-2 i t}-1}{-2 i}-t\right)+R_{3},
\end{aligned}
$$

with the bounded remainder term

$$
R_{3}:=N\left(\frac{e^{(-4 N+6) i t}-1}{4 N-6}-\frac{e^{(-4 N+8) i t}-1}{4 N-8}\right)
$$

which fulfills $\left|R_{3}\right| \leq 1$.

The last contribution to $N^{s} e^{\mathcal{H} \widehat{\partial_{x}^{2} t} I_{2,2}}(t)(N)$ comes from $\widehat{I_{2}\left(t^{\prime}\right)}(N+2)$ and $\widehat{I_{2}\left(t^{\prime}\right)}(-2)$. As above, using $-N^{2}-4+(N+2)^{2}-4 N=0$, we calculate

$$
\begin{aligned}
J_{4} & :=-i(N+2) \int_{0}^{t} e^{-i t^{\prime} N^{2}} e^{-4 i t^{\prime}}\left(e^{2 i t^{\prime}}-1\right) e^{i t^{\prime}(N+2)^{2}}\left(e^{-4 N i t^{\prime}}-1\right) d t^{\prime} \\
& =-i(N+2)\left(\int_{0}^{t}\left(e^{2 i t^{\prime}}-1\right) d t^{\prime}-\int_{0}^{t} e^{4 N i t^{\prime}}\left(e^{2 i t^{\prime}}-1\right) d t^{\prime}\right) \\
& =-i(N+2)\left(\frac{e^{2 i t}-1}{2 i}-t\right)+R_{4}
\end{aligned}
$$

with the bounded remainder term

$$
R_{4}:=(N+2)\left(\frac{e^{(4 N+2) i t}-1}{4 N+2}-\frac{e^{4 N i t}-1}{4 N}\right),
$$

which satisfies $\left|R_{4}\right| \leq 1$.

Summing up all contributions to $N^{s} e^{\mathcal{H} \partial_{x}^{2} t I_{2,2}}(t)(N)$ we arrive at

$$
\left\|I_{2,2}(t)\right\|_{H^{s}(\mathbb{T})} \geq\left|J_{1}+J_{2}+J_{3}+J_{4}\right|
$$

according to (2.2). With the complex number

$$
z(t):=-i\left(\frac{e^{2 i t}-1}{2 i}-t\right)
$$


we have

$$
\left|J_{1}+J_{2}+J_{3}+J_{4}\right| \geq(6 N+4)|\operatorname{Re} z(t)|-12 .
$$

Finally, we observe that $\operatorname{Re} z(t)=\sin ^{2}(t)$.

Proof of Theorem 1.1. Let $s \in \mathbb{R}, T>0$. Assume that there exists a constant $c>0$ and a normed space $X_{T}$, which is continuously embedded in $C\left([0, T], \dot{H}^{s}(\mathbb{T})\right)$ with the properties (1.2), (1.3), and define $u_{N}=I_{2}\left(\psi_{N}\right)$. Then, using the estimate (1.3) twice, followed by one application of (1.2), we have

$$
\begin{aligned}
\left\|\int_{0}^{t} e^{\mathcal{H} \partial_{x}^{2}\left(t-t^{\prime}\right)} \partial_{x}\left(u_{N}^{2}\left(t^{\prime}\right)\right) d t^{\prime}\right\|_{X_{T}} & \leq c\left\|u_{N}\right\|_{X_{T}}^{2} \leq c^{3}\left\|e^{\mathcal{H} \partial_{x}^{2} t} \psi_{N}\right\|_{X_{T}}^{4} \\
& \leq c^{7}\left\|\psi_{N}\right\|_{H^{s}(\mathbb{T})}^{4} \leq c^{7} .
\end{aligned}
$$

On the other hand, because of the continuous embedding, there exists $d>0$ such that the left-hand side is bounded from below by

$$
d\left\|\int_{0}^{t} e^{\mathcal{H} \partial_{x}^{2}\left(t-t^{\prime}\right)} \partial_{x}\left(u_{N}^{2}\left(t^{\prime}\right)\right) d t^{\prime}\right\|_{H^{s}(\mathbb{T})}=d\left\|I_{2,2}(t)\right\|_{H^{s}(\mathbb{T})} \geq d N \sin ^{2}(t)-12 d
$$

for any $N \in \mathbb{N}, t \in[0, T]$, which is a contradiction.

\section{REFERENCES}

1. Jean Bourgain, Fourier transform restriction phenomena for certain lattice subsets and applications to nonlinear evolution equations. II. The KdV-equation, Geom. Funct. Anal. 3 (1993), no. 3, 209-262. MR 1215780 (95d:35160b)

2. Sebastian Herr, Well-posedness for equations of Benjamin-Ono type, Illinois J. Math. 51 (2007), no. 3, 951-976.

3. Alexandru D. Ionescu and Carlos E. Kenig, Global well-posedness of the Benjamin-Ono equation in low-regularity spaces, J. Amer. Math. Soc. 20 (2007), 753-798. MR.2291918

4. _ Complex-valued solutions of the Benjamin-Ono equation. In Harmonic Analysis, Partial Differential Equations, and Related Topics, Contemp. Math., vol. 428, Amer. Math. Soc., Providence, RI, 2007, pp. 61-74. MR.2322378

5. Carlos E. Kenig, Gustavo Ponce, and Luis Vega, Well-posedness and scattering results for the generalized Korteweg-de Vries equation via the contraction principle, Comm. Pure Appl. Math. 46 (1993), no. 4, 527-620. MR1211741 (94h:35229)

6. — A bilinear estimate with applications to the KdV equation, J. Amer. Math. Soc. 9 (1996), no. 2, 573-603. MR.1329387 (96k:35159)

7. Herbert Koch and Nikolay Tzvetkov, Nonlinear wave interactions for the Benjamin-Ono equation, Int. Math. Res. Not. (2005), no. 30, 1833-1847. MR2172940 (2006f:35245)

8. Luc Molinet, Jean-Claude Saut, and Nikolay Tzvetkov, Ill-posedness issues for the BenjaminOno and related equations, SIAM J. Math. Anal. 33 (2001), no. 4, 982-988 (electronic). MR:1885293 (2002k:35281)

9. Luc Molinet, Global well-posedness in the energy space for the Benjamin-Ono equation on the circle, Math. Ann. 337 (2007), no. 2, 353-383. MR2262788

10. Global well-posedness in $L^{2}$ for the periodic Benjamin-Ono equation, to appear in Amer. J. Math., arXiv:math.AP/0601217

11. Terence Tao, Global well-posedness of the Benjamin-Ono equation in $H^{1}(\mathbf{R})$, J. Hyperbolic Differ. Equ. 1 (2004), no. 1, 27-49. MR2052470 (2005f:35273)

Technische Universität Dortmund, Fakultät für Mathematik, 44221 Dortmund, GerMANY

Current address: Center for Pure and Applied Mathematics, University of California, 837

Evans Hall, Berkeley, California 94720-3840

E-mail address: herr@math.berkeley.edu 